\title{
ON THE UNIQUENESS OF ASYMPTOTIC SOLUTIONS OF LINEAR DIFFERENTIAL EQUATIONS
}

\author{
F. W. J. Olver \\ Dedicated to Richard Askey on the occasion of his 65th birthday and \\ in celebration of his many contributions to classical analysis
}

\begin{abstract}
We consider the characterization of solutions of a linear differential equation of arbitrary order in the neighborhood of an irregular singularity of rank unity, on the assumption that the characteristic values are distinct. We show that some solutions can be defined uniquely simply by their asymptotic behavior along a single ray, whereas for other solutions the asymptotic behavior must be maintained along two rays to ensure uniqueness. The results are needed in the construction of algorithms for the computation of solutions.
\end{abstract}

\section{Introduction}

We consider solutions of the differential equation

$$
\frac{d^{n} w}{d z^{n}}+f_{n-1}(z) \frac{d^{n-1} w}{d z^{n-1}}+f_{n-2}(z) \frac{d^{n-2} w}{d z^{n-2}}+\cdots+f_{0}(z) w=0
$$

in the neighborhood of the point at infinity, on the assumptions that $n \geq 2$ and the coefficients $f_{\ell}(z), \ell=0,1, \ldots, n-1$, are analytic at infinity, that is, they can be expanded in power series of the form

$$
f_{\ell}(z)=\sum_{s=0}^{\infty} \frac{f_{s \ell}}{z^{s}}
$$

that converge for all sufficiently large values of $|z|$. Thus, the singularity of equation (1.1) at infinity is no worse than an irregular singularity of rank 1.

Formal solutions of (1.1) are given by

$$
e^{\lambda_{j} z} z^{\mu_{j}} \sum_{s=0}^{\infty} \frac{a_{s j}}{z^{s}}, \quad j=1,2, \ldots, n,
$$

with $a_{0 j}=1$. The characteristic values $\lambda_{j}$, indices $\mu_{j}$, and remaining coefficients $a_{s j}$ are obtained by substituting into (1.1) by means of (1.2), (1.3), and the differentiated forms of (1.3), and equating coefficients. We shall restrict our attention to the case in which the $\lambda_{j}$ are distinct; however, the main conclusions in this paper will carry over to more general situations.

Received May 8, 1998, revised August 17, 1998.

1991 Mathematics Subject Classification: 34E05.

Key words and phrases: anti-Stokes lines, asymptotic expansions, differential equations, equidominant solutions, explicit solutions, implicit solutions, recessive solutions, singularities, uniqueness. 
In order to describe the sectors in which the formal solutions furnish asymptotic expansions of actual solutions for large $z$, we denote

$$
\theta_{j k}=\operatorname{ph}\left(\lambda_{k}-\lambda_{j}\right), \quad k \neq j
$$

resolving the ambiguity in the choice of these phase angles by requiring $\theta_{j k}$ to lie in a prescribed, but arbitrary, half-closed interval $\mathcal{I}$ of length $2 \pi$. Next, we define $\mathcal{S}_{j}(\mathcal{I})$ to be the open sector

$$
\mathcal{S}_{j}(\mathcal{I})=\left\{z:-\frac{3}{2} \pi-\alpha_{j}<\operatorname{ph} z<\frac{3}{2} \pi-\beta_{j}\right\}
$$

in which

$$
\alpha_{j}=\min _{k \neq j} \theta_{j k}, \quad \beta_{j}=\max _{k \neq j} \theta_{j k}
$$

Theorem 1.1. For each $j=1,2, \ldots, n$, there exists a unique solution $w_{j}(\mathcal{I} \mid z)$ of (1.1) with the property

$$
w_{j}(\mathcal{I} \mid z) \sim e^{\lambda_{j} z} z^{\mu_{j}} \sum_{s=0}^{\infty} \frac{a_{s j}}{z^{s}}
$$

as $z \rightarrow \infty$, uniformly in any closed sector properly interior to $\mathcal{S}_{j}(\mathcal{I})$. This asymptotic expansion can be differentiated $n-1$ times in the same circumstances, and the $n$ solutions $w_{j}(\mathcal{I} \mid z)$ are linearly independent. Furthermore, the sectors of validity $\mathcal{S}_{j}(\mathcal{I})$ are maximal, in general.

This theorem is proved in [5], and this reference includes strict bounds for the remainder terms in the expansion (1.7) and its differentiated forms.

The conditions of Theorem 1.1 (and also Theorems 2.1 and 2.2 below) require the property (1.7) to hold uniformly on sectorial domains in order to ensure the uniqueness of the solution $w_{j}(\mathcal{I} \mid z)$. The purpose of the present paper is to simplify this condition. We seek unbounded point sets $\mathcal{T}$ in $\mathbb{C}$, for example, rays, such that if $w(z)$ is a solution of (1.1) with either the property

$$
w(z) \sim e^{\lambda_{j} z} z^{\mu_{j}}, \quad z \rightarrow \infty \quad \text { on } \mathcal{T}
$$

or the stronger property

$$
w(z) \sim e^{\lambda_{j} z} z^{\mu_{j}} \sum_{s=0}^{\infty} \frac{a_{s j}}{z^{s}}, \quad z \rightarrow \infty \quad \text { on } \mathcal{T},
$$

then

$$
w(z)=w_{j}(\mathcal{I} \mid z)
$$

Another way of characterizing solutions uniquely is by their hyperasymptotic behavior along a single ray [2]. The advantage of the present approach is that it provides a basis for the boundary-value methods needed in the numerical computation of the solutions anywhere in $\mathbb{C}$, not merely in the neighborhood of the singular point; see [3]. 


\section{Preliminary results}

The common region of validity of the expansion (1.7) for $j=1,2, \ldots, n$ is given by

$$
\mathcal{S}(\mathcal{I})=\mathcal{S}_{1}(\mathcal{I}) \cap \mathcal{S}_{2}(\mathcal{I}) \cap \cdots \cap \mathcal{S}_{n}(\mathcal{I})
$$

that is,

$$
\mathcal{S}(\mathcal{I})=\left\{z:-\frac{3}{2} \pi-\alpha<\operatorname{ph} z<\frac{3}{2} \pi-\beta\right\}
$$

where

$$
\alpha=\min _{k \neq j} \theta_{j k}, \quad \beta=\max _{k \neq j} \theta_{j k},
$$

the minimum and maximum now being taken over all possible values of $j$ and $k$.

Theorem 2.1. There exist linearly independent and unique solutions $w_{j}(\mathcal{I} \mid z), j=$ $1,2, \ldots, n$, such that (1.7) applies as $z \rightarrow \infty$, uniformly in any closed sector properly interior to $\mathcal{S}(\mathcal{I})$.

This theorem follows immediately from Theorem 1.1, except for the uniqueness property associated with the smaller sector $\mathcal{S}(\mathcal{I})$. The proof of this property was supplied in $[5, \S 10]$.

The following results were stated in $[3, \S 2]$ and also appear in earlier literature. (See [1] for Theorem 2.2 and [6, Chapter 4] for Theorem 2.3.) Since they are important in subsequent analysis, we include brief proofs here for completeness. As usual, by an "anti-Stokes line", we mean a ray on which $\theta_{p q}+\operatorname{ph} z$ is an odd multiple of $\frac{1}{2} \pi$ for at least one unequal pair of values of $p$ and $q$.

Theorem 2.2. Let $\mathcal{Z}$ be an arbitrary closed sector of angle $\pi$ whose boundaries do not coincide with anti-Stokes lines. Then there is a set of linearly independent and unique solutions $w_{j}(z), j=1,2, \ldots, n$, such that

$$
w_{j}(z) \sim e^{\lambda_{j} z} z^{\mu_{j}} \sum_{s=0}^{\infty} \frac{a_{s j}}{z^{s}}
$$

uniformly as $z \rightarrow \infty$ in $\mathcal{Z}$.

Theorem 2.3. Let $\mathcal{Z}$ be an arbitrary ray or an arbitrary closed sector of angle less than $\pi$. Then there is a set of linearly independent (but not necessarily unique) solutions $w_{j}(z), j=1,2, \ldots, n$, such that (2.4) applies uniformly as $z \rightarrow \infty$ in $\mathcal{Z}$.

Proof of Theorem 2.2. Let $\mathcal{Z}$ be bounded by the rays ph $z=\phi-\pi$ and ph $z=\phi$. In Theorem 2.1, take $\mathcal{I}=\left(-\frac{1}{2} \pi-\phi, \frac{3}{2} \pi-\phi\right]$. Then it is easily verified that $-\frac{3}{2} \pi-\alpha+\delta \leq$ $\phi-\pi$ and $\frac{3}{2} \pi-\beta-\delta \geq \phi$ where $\alpha$ and $\beta$ are defined by (2.3) and $\delta$ is the smaller of

$$
\min _{k \neq j}\left(\theta_{j k}+\frac{1}{2} \pi+\phi\right), \quad \min _{k \neq j}\left(\frac{3}{2} \pi-\phi-\theta_{j k}\right)
$$

the minima being taken over all possible values of $j$ and $k$. Because $\theta_{j k} \epsilon$ $\left(-\frac{1}{2} \pi-\phi, \frac{3}{2} \pi-\phi\right]$ and $\theta_{j k}+\phi$ cannot be an odd multiple of $\frac{1}{2} \pi, \delta$ is necessarily positive. Accordingly $\mathcal{Z} \subset \mathcal{S}(\mathcal{I})$, and the existence of the $w_{j}(z)$ follows from Theorem 2.1. The uniqueness property is proved again as in $[5, \S 10]$. 
The condition that the boundaries of $\mathcal{Z}$ are not anti-Stokes lines cannot be omitted from Theorem 2.2. An example is provided by Bessel's equation

$$
\frac{d^{2} w}{d z^{2}}+\frac{1}{z} \frac{d w}{d z}+\left(1-\frac{\nu^{2}}{z^{2}}\right) w=0
$$

Here $n=2, \lambda_{1}=i, \lambda_{2}=-i, \mu_{1}=\mu_{2}=-\frac{1}{2}$, and the anti-Stokes lines are the positive and negative parts of the real axis. Using the well-known properties of the solutions $H_{\nu}^{(1)}(z)$ and $H_{\nu}^{(2)}(z)$ of this equation [4, Chapter 7], we can prove, for example, that no solution exists that is asymptotic to $e^{i z} z^{-1 / 2}$ uniformly in the sector $-\pi \leq \mathrm{ph}$ $z \leq 0$, except when the parameter $\nu$ is half an odd integer. ${ }^{1}$

Proof of Theorem 2.3. Theorem 2.3 is an immediate consequence of Theorem 2.2, inasmuch as any closed sector of angle less than $\pi$ is always properly interior to a sector of angle $\pi$ whose boundaries are not anti-Stokes lines.

Theorem 2.3 also can be deduced directly from Theorem 2.1, as follows. Let the boundaries of $\mathcal{Z}$ be the rays ph $z=\phi_{1}$ and ph $z=\phi_{2}$, with $0 \leq \phi_{2}-\phi_{1}<\pi$. (By permitting $\phi_{2}-\phi_{1}=0$, we include the case in which $\mathcal{Z}$ is a ray.) In Theorem 2.1 , take $\mathcal{I}=\left(-\pi-\frac{1}{2} \phi_{1}-\frac{1}{2} \phi_{2}, \pi-\frac{1}{2} \phi_{1}-\frac{1}{2} \phi_{2}\right]$. Then it is easily verified that $\mathcal{Z}$ is properly interior to $\mathcal{S}(\mathcal{I})$, and the result follows. (The uniqueness property now has been sacrificed.)

The final topic in this section is the relative behavior, as $z \rightarrow \infty$, of any two distinct members $w_{j}(z)$ and $w_{k}(z)$ of the set of solutions furnished by Theorem 2.3 in the case when $\mathcal{Z}$ is a single ray $\mathfrak{L}$, given by

$$
\mathfrak{L}=\left\{z: z=t e^{i \theta}, 0<t<\infty\right\} .
$$

We have three cases to consider:

(i) $\operatorname{Re}\left\{\left(\lambda_{k}-\lambda_{j}\right) z\right\} \neq 0$ on $\mathfrak{L}$,

(ii) $\operatorname{Re}\left\{\left(\lambda_{k}-\lambda_{j}\right) z\right\}=0$ on $\mathfrak{L}$, and $\operatorname{Re} \mu_{k} \neq \operatorname{Re} \mu_{j}$,

(iii) $\operatorname{Re}\left\{\left(\lambda_{k}-\lambda_{j}\right) z\right\}=0$ on $\mathfrak{L}$, and $\operatorname{Re} \mu_{k}=\operatorname{Re} \mu_{j}$.

In Case (i), if, for example, $\operatorname{Re}\left\{\left(\lambda_{k}-\lambda_{j}\right) z\right\}$ is negative on $\mathfrak{L}$, then $w_{k}(z) / w_{j}(z) \rightarrow 0$ as $z \rightarrow \infty$, and we say that $w_{j}(z)$ dominates $w_{k}(z)$ exponentially as $z \rightarrow \infty$ on $\mathfrak{L}$. Similarly in Case (ii), if, for example, $\operatorname{Re}\left(\mu_{k}-\mu_{j}\right)$ is negative, then again $w_{k}(z) / w_{j}(z) \rightarrow 0$ as $z \rightarrow \infty$, but this time we say that $w_{j}(z)$ dominates $w_{k}(z)$ algebraically as $z \rightarrow \infty$ on $\mathfrak{L}$. Lastly, in Case (iii), $\left|w_{k}(z) / w_{j}(z)\right| \rightarrow$ a nonzero constant as $z \rightarrow \infty$, and we say that $w_{j}(z)$ and $w_{k}(z)$ are equidominant on $\mathfrak{L}$.

Uniqueness problems are not difficult to resolve when only exponential dominance is involved. To handle other cases, we shall make frequent use of the following result:

Lemma. On a given ray $\mathfrak{L}$, no nontrivial linear combination of a set of equidominant solutions can be dominated by a member of the set.

\footnotetext{
${ }^{1}$ This is a consequence of the Stokes phenomenon. When the Stokes ray ph $z=-\frac{1}{2} \pi$ is crossed, a multiple of the solution that is exponentially small on that ray is switched on. This multiple vanishes iff $2 \nu$ is an odd integer.
} 
Proof. Let the solutions be enumerated in such a way that the equidominant set is $w_{p}(z), w_{p+1}(z), \ldots, w_{q}(z), p$ and $q$ being integers such that $1 \leq p<q \leq n$, and let

$$
w(z)=A_{p} w_{p}(z)+A_{p+1} w_{p+1}(z)+\cdots+A_{q} w_{q}(z)
$$

where the $A$ 's are constants. Let us assume that

$$
w(z)=o\left\{w_{k}(z)\right\}, \quad z \rightarrow \infty \text { on } \mathfrak{L},
$$

where $p \leq k \leq q$. Then we have to prove that $A_{p}=A_{p+1}=\cdots=A_{q}=0$.

By hypothesis,

$$
\lambda_{j} e^{i \theta}=a+i b_{j}, \quad \mu_{j}=c+i d_{j}, \quad j=p, p+1, \ldots, q,
$$

where $a, b_{j}, c$, and $d_{j}$ are real constants, $a$ and $c$ being independent of $j$, and the $b_{j}$ being unequal. Hence, on $\mathfrak{L}$,

$$
e^{\lambda_{j} z} z^{\mu_{j}}=e^{\left(a+i b_{j}\right) t} t^{c+i d_{j}} e^{i \theta c-\theta d_{j}}
$$

On replacing the $w$ 's in (2.6) and (2.7) by means of their limiting forms, and then substituting by means of $(2.8)$, we find that

$$
\sum_{j=p}^{q} \hat{A}_{j} e^{i b_{j} t} t^{i d_{j}}=o(1)
$$

as $t \rightarrow \infty$, where

$$
\hat{A}_{j}=e^{-\theta d_{j}} A_{j}, \quad j=p, p+1, \ldots, q .
$$

Now let $\sigma$ be an arbitrary constant such that

$$
0<\sigma<\frac{2 \pi}{\max \left|b_{j}-b_{k}\right|}
$$

the maximum being taken over $j, k \in[p, q]$. In (2.9), set $t=(\tau+s) \sigma$ with $s=$ $0,1, \ldots, q-p$ in turn, and then let $\tau \rightarrow \infty$. Since

$$
t^{i d_{j}}=\{(\tau+s) \sigma\}^{i d_{j}}=(\sigma \tau)^{i d_{j}}\left\{1+O\left(\tau^{-1}\right)\right\}
$$

uniformly for $s=0,1, \ldots, q-p$, we have

$$
\begin{aligned}
\sum_{j=p}^{q} \hat{A}_{j} e^{i b_{j} \sigma \tau}(\sigma \tau)^{i d_{j}} & =o(1), \\
\sum_{j=p}^{q} \hat{A}_{j} e^{i b_{j} \sigma \tau+i b_{j} \sigma}(\sigma \tau)^{i d_{j}} & =o(1), \\
\vdots & \\
\sum_{j=p}^{q} \hat{A}_{j} e^{i b_{j} \sigma \tau+i(q-p) b_{j} \sigma}(\sigma \tau)^{i d_{j}} & =o(1) .
\end{aligned}
$$

This is a system of $q-p+1$ inhomogeneous equations for the $q-p+1$ unknowns $\hat{A}_{p}$, $\hat{A}_{p+1}, \ldots, \hat{A}_{q}$. The determinant $D$, say, of this system is given by

$$
D=e^{i\left(b_{p}+\cdots+b_{q}\right) \sigma \tau}(\sigma \tau)^{i\left(d_{p}+\cdots+d_{q}\right)} \prod_{p \leq j<k \leq q}\left(e^{i b_{k} \sigma}-e^{i b_{j} \sigma}\right) .
$$


Clearly $|D|$ is independent of $\tau$, and in consequence of (2.11) and the fact that $b_{j} \neq b_{k}$ when $j \neq k,|D|$ does not vanish. Applying Cramer's rule and letting $\tau \rightarrow \infty$, we deduce that $\hat{A}_{p}=\hat{A}_{p+1}=\cdots=\hat{A}_{q}=0$, and, hence from (2.10), $A_{p}=A_{p+1}=\cdots=$ $A_{q}=0$.

\section{Explicit solutions}

In [3], new classifications of the asymptotic solutions given by Theorem 1.1 were introduced as follows. If $\beta_{j}-\alpha_{j} \in[0, \pi)$, then the corresponding solution $w_{j}(\mathcal{I} \mid z)$ is said to be explicit. Alternatively, when $\beta_{j}-\alpha_{j} \in[\pi, 2 \pi), w_{j}(\mathcal{I}|z|)$ is said to be implicit. In the explicit case, $\mathcal{S}_{j}(\mathcal{I})$ contains the nonempty open sector

$$
\mathcal{R}_{j}(\mathcal{I})=\left\{z:-\frac{1}{2} \pi-\alpha_{j}<\operatorname{ph} z<\frac{1}{2} \pi-\beta_{j}\right\} .
$$

This is called the recessive sector for $w_{j}(\mathcal{I} \mid z)$, because if $z \rightarrow \infty$ along any chosen ray $\mathfrak{L}$ in $\mathcal{R}_{j}(\mathcal{I})$, then $w_{j}(\mathcal{I} \mid z)$ is dominated, indeed dominated exponentially, by any solution with asymptotic form $e^{\lambda_{k} z} z^{\mu_{k}}(k \neq j)$ on $\mathfrak{L}$.

When $n=2$, we have $\beta_{j}=\alpha_{j}$; hence, both asymptotic solutions are explicit, and it is easily seen (and already well known) that $w(z)$ is specified uniquely by (1.8a) with $\mathcal{T}=\mathfrak{L}$, provided again that $\mathfrak{L} \subset \mathcal{R}_{j}(\mathcal{I})$. We begin this section by proving that this result holds for explicit solutions for all values of $n$.

To simplify notation, we may suppose that $j=1$, so that (1.8a) becomes

$$
w(z) \sim e^{\lambda_{1} z} z^{\mu_{1}}, \quad z \rightarrow \infty \text { on } \mathfrak{L},
$$

with $\mathfrak{L} \subset \mathcal{R}_{1}(\mathcal{I})$. Also, we let $w_{1}(z), w_{2}(z), \ldots, w_{n}(z)$ denote solutions furnished by Theorem 2.3 with $\mathcal{Z}=\mathfrak{L}$. Then constants $A_{1}, A_{2}, \ldots, A_{n}$ exist such that

$$
w(z)=A_{1} w_{1}(z)+A_{2} w_{2}(z)+\cdots+A_{n} w_{n}(z) .
$$

Suppose first that none of the solutions are equidominant on $\mathfrak{L}$. Then they may be ranked in order of dominance, and by relabelling $w_{2}(z), w_{3}(z), \ldots, w_{n}(z)$, if necessary, we may suppose that the ascending order of rank is given by $w_{1}(z), w_{2}(z), \ldots, w_{n}(z)$. On dividing (3.3) by $w_{n}(z)$, letting $z \rightarrow \infty$ on $\mathfrak{L}$ and using (3.2), we see immediately that $A_{n}=0$. Similarly, $A_{n-1}=A_{n-2}=\cdots=A_{2}=0$, and $A_{1}=1$. Since $w_{1}(\mathcal{I} \mid z)$ satisfies (3.2), it follows that any solution that satisfies (3.2) must be the same as $w_{1}(\mathcal{I} \mid z)$.

In the general case, we group the sets of equidominant solutions in blocks when we arrange the solutions $w_{1}(z), w_{2}(z), \ldots, w_{n}(z)$ in ascending order of dominance on $\mathfrak{L}$. If we follow the previous method of proof, and $w_{p}(z), w_{p+1}(z), \ldots, w_{q}(z)$ is the first block of equidominant solutions that we encounter, then we have $1<p<q \leq n$ and

$$
A_{p} w_{p}(z)+A_{p+1} w_{p+1}(z)+\cdots+A_{q} w_{q}(z)=o\left\{w_{p}(z)\right\}
$$

as $z \rightarrow \infty$ on $\mathfrak{L}$. Accordingly, from the Lemma of $\S 2, A_{p}=A_{p+1}=\cdots=A_{q}=0$. Similarly for the other blocks, and the remainder of the proof is completed as before. Therefore, we have proved the following result:

Theorem 3.1. Let $\mathfrak{L}$ be any ray within the recessive sector $\mathcal{R}_{j}(\mathcal{I})$ of an explicit solution $w_{j}(\mathcal{I} \mid z)$ of (1.1). Then condition (1.8a), with $\mathcal{T}=\mathfrak{L}$, implies (1.9).

If we substitute $(1.8 \mathrm{~b})$ for $(1.8 \mathrm{a})$, then we may permit $\mathfrak{L}$ to coincide with a boundary of $\mathcal{R}_{j}(\mathcal{I})$ : 
Theorem 3.2. Let $\mathcal{L}$ be any ray in the closure of the recessive sector $\mathcal{R}_{j}(\mathcal{I})$. Then condition (1.8b), with $\mathcal{T}=\mathfrak{L}$, implies (1.9).

Proof. The difference in the proof of this result from that of Theorem 3.1 stems from the possibility that if $\mathfrak{L}$ coincides with a boundary of $\mathcal{R}_{1}(\mathcal{I})$, then there are members of the set $w_{2}(z), w_{3}(z), \ldots, w_{n}(z)$ that are equidominant with, or dominated algebraically by, $w_{1}(z)$ as $z \rightarrow \infty$ on $\mathfrak{L}$. Let the latter set be $w_{2}(z), w_{3}(z), \ldots, w_{p}(z)$, where $1 \leq p \leq$ $n$, and assume that in nondescending order of dominance the complete set of solutions is given by

$$
w_{2}(z), w_{3}(z), \ldots, w_{p}(z), w_{1}(z), w_{p+1}(z), w_{p+2}(z), \ldots, w_{n}(z) .
$$

On following the proof of Theorem 3.1, we first conclude that equation (3.3) reduces to

$$
w(z)=A_{2} w_{2}(z)+\cdots+A_{p} w_{p}(z)+A_{1} w_{1}(z)+A_{p+1} w_{p+1}(z)+\cdots+A_{q} w_{q}(z)
$$

where $p \leq q \leq n$, and the solutions $w_{1}(z), w_{p+1}(z), \ldots, w_{q}(z)$ are equidominant on $\mathfrak{L}$. Hence, on using the assumption (1.8b) with $j=1$, we have

$$
\left(A_{1}-1\right) w_{1}(z)+A_{p+1} w_{p+1}(z)+\cdots+A_{q} w_{q}(z)=o\left\{w_{1}(z)\right\}
$$

as $z \rightarrow \infty$ on $\mathfrak{L}$. Application of the Lemma of $\S 2$ then shows that $A_{1}-1=A_{p+1}=$ $\cdots=A_{q}=0$, so that (3.4) reduces to

$$
A_{2} w_{2}(z)+\cdots+A_{p} w_{p}(z)=w(z)-w_{1}(z)
$$

Again, from (2.4) and (1.8b) with $j=1$ in both cases, it follows that the right-hand side of (3.5) is $O\left(e^{\lambda_{1} z} z^{\mu_{1}-m}\right)$ where $m$ is an arbitrary integer. This estimate may be included in $o\left\{w_{2}(z)\right\}$ by choosing $m$ large enough, and proceeding again as in the proof of Theorem 3.1, we conclude that $A_{2}=A_{3}=\cdots=A_{p}=0$.

\section{Implicit solutions}

Unlike explicit solutions, in general it is not possible to characterize an implicit solution $w_{j}(\mathcal{I} \mid z)$ by its asymptotic behavior along a single ray $\mathcal{L}$. This is because, for all choices of $\mathcal{L}$ in the sector $\mathcal{S}_{j}(\mathcal{I})$ of asymptotic validity, there is always a nontrivial solution of (1.1) that is dominated exponentially by $w_{j}(\mathcal{I} \mid z)$ as $z \rightarrow \infty$, the only possible exception arising when $\beta_{j}-\alpha_{j}=\pi[3, \S 3]$. However, two rays, suitably restricted, always suffice:

Theorem 4.1. Let $\mathcal{Z}$ be a nondegenerate closed sector within $\mathcal{S}_{j}(\mathcal{I})$ with boundaries $\mathfrak{L}$ and $\mathfrak{L}^{\prime}$. Assume that either: (i) $\mathcal{Z}$ is of angle less than $\pi$; or (ii) the angle of $\mathcal{Z}$ is $\pi$ and neither $\mathfrak{L}$ nor $\mathfrak{L}^{\prime}$ is an anti-Stokes line of (1.1). Assume also that in Case (i), no nontrivial solution of $(1.1)$ is dominated exponentially by $w_{j}(\mathcal{I} \mid z)$ on both $\mathfrak{L}$ and $\mathfrak{L}^{\prime}$ as $z \rightarrow \infty$. Then condition (1.8b), with $\mathcal{T}=\mathfrak{L} \cup \mathfrak{L}^{\prime}$, implies (1.9). 
Proof. The proof of Theorem 4.1 is similar to the proofs of Theorem 3.1 and 3.2, and proceeds as follows. With the given conditions, there is a set of linearly independent solutions $w_{k}(z), k=1,2, \ldots, n$, such that

$$
w_{k}(z) \sim e^{\lambda_{k} z} z^{\mu_{k}} \sum_{s=0}^{\infty} \frac{a_{s k}}{z^{s}}
$$

as $z \rightarrow \infty$ on both $\mathfrak{L}$ and $\mathfrak{L}^{\prime}$. This is a consequence of Theorem 2.3 in Case (i) and Theorem 2.2 in Case (ii). We may express $w(z)$ in the form

$$
w(z)=A_{1} w_{1}(z)+A_{2} w_{2}(z)+\cdots+A_{n} w_{n}(z)
$$

in which the $A$ 's are constants. If we let $z \rightarrow \infty$ on $\mathfrak{L}$, then it follows that $A_{k}=0$ for those values of $k(\neq j)$ for which $w_{k}(z)$ dominates, or is equidominant with, or is algebraically dominated by, $w_{j}(z)$ on $\mathcal{L}$, and $A_{j}=1$. Similarly, $A_{k}=0$ for those values of $k(\neq j)$ for which $w_{k}(z)$ dominates, or is equidominant with, or is algebraically dominated by, $w_{j}(z)$ on $\mathfrak{L}^{\prime}$ (and $A_{j}=1$ ). In both Cases (i) and (ii), only the term $w_{j}(z)$ survives on the right-hand side of (4.1). And since $w_{j}(\mathcal{I} \mid z)$ satisfies $(1.8 \mathrm{~b})$ with $\mathcal{T}=\mathfrak{L} \cup \mathfrak{L}^{\prime}$, it follows that (1.9) applies.

It should be observed that Theorem 4.1 actually applies to any of the solutions $w_{j}(\mathcal{I} \mid z)$. If $w_{j}(\mathcal{I} \mid z)$ is explicit, then the theorem provides a way of specifying $w_{j}(\mathcal{I} \mid z)$ uniquely by its asymptotic behavior on two rays that lie outside its recessive sector.

There is one case in which an implicit solution can be characterized by its asymptotic behavior along a single ray:

Theorem 4.2. Assume that $\beta_{j}-\alpha_{j}=\pi$ and

$$
\mathfrak{L}=\left\{z: \operatorname{ph} z=-\frac{1}{2} \pi-\alpha_{j}\left(=\frac{1}{2} \pi-\beta_{j}\right)\right\}
$$

Then condition (1.8b), with $\mathcal{T}=\mathfrak{L}$, implies (1.9).

The proof of Theorem 4.2 is essentially the same as that of Theorem 3.2. In fact, Theorem 4.2 may be regarded as a limiting form of Theorem 3.2 because, as $\beta_{j}-\alpha_{j} \rightarrow \pi$, the boundaries of the recessive sector $\mathcal{R}_{j}(\mathcal{I})$ coalesce into $\mathfrak{L}$.

\section{Examples}

We first note that the conditions of Theorem 4.1 always can be satisfied by taking $\mathfrak{L}$ and $\mathfrak{L}^{\prime}$ to be within $\mathcal{S}_{j}(\mathcal{I})$ and the images of each other in the origin, provided that they are not anti-Stokes lines. For numerical purposes, however, it is sometimes desirable to locate $\mathfrak{L}$ and $\mathfrak{L}^{\prime}$ instead along anti-Stokes lines. 
Thus, in $[3, \S 8.2]$, to compute an implicit solution $w_{1}\left(\mathcal{I}_{1} \mid z\right)$ of a certain fourth-order differential equation, rays $\mathfrak{L}$ and $\mathfrak{L}^{\prime}$ were used along anti-Stokes lines as depicted in Figure 8.5 of this reference. On $\mathfrak{L}$, only multiples of the solution $w_{4}(z)$ are dominated exponentially by $w_{1}(z)$, and on $\mathfrak{L}^{\prime}$, only multiples of $w_{3}(z)$ are dominated exponentially by $w_{1}(z)$. In consequence, the conditions of Theorem 4.1 (Case(i)) of the present paper are satisfied; accordingly, $w_{1}\left(\mathcal{I}_{1} \mid z\right)$ is defined uniquely by its asymptotic behavior on $\mathfrak{L}$ and $\mathfrak{L}^{\prime}$. This is the underlying reason that in [3], we were able to place the boundary values for computing $w_{1}\left(\mathcal{I}_{1} \mid z\right)$ on $\mathfrak{L}$ and $\mathfrak{L}^{\prime}$. (Even so, as in [3], perturbation analysis is needed to determine precisely how the $n$ necessary boundary values must be distributed on $\mathfrak{L} \cup \mathfrak{L}^{\prime}$ to ensure stability in the computation of the wanted solution.)

Another interesting situation is to suppose that all the $\lambda$ 's lie on the imaginary axis (and are, of course, distinct). An example is provided by the differential equation

$$
\frac{d^{5} w}{d z^{5}}+\left(\frac{4}{z}-\frac{1}{z^{3}}\right) \frac{d^{4} w}{d z^{4}}+5 \frac{d^{3} w}{d z^{3}}+\frac{7}{z} \frac{d^{2} w}{d z^{2}}+4 \frac{d w}{d z}+\left(\sin \frac{1}{z}\right)^{2} w=0
$$

Here, $\lambda_{1}=-2 i, \lambda_{2}=-i, \lambda_{3}=0, \lambda_{4}=i, \lambda_{5}=2 i$; correspondingly, $\mu_{1}=-\frac{3}{2}$, $\mu_{2}=-\frac{1}{2}, \mu_{3}=0, \mu_{4}=-\frac{1}{2}, \mu_{5}=-\frac{3}{2}$. Let us take $\mathcal{I}=(-\pi, \pi]$. Then, for the extreme values of $\lambda$, we have, from (1.6), $\alpha_{1}=\beta_{1}=\frac{1}{2} \pi$ and $\alpha_{5}=\beta_{5}=-\frac{1}{2} \pi$. Accordingly, the corresponding solutions $w_{1}(\mathcal{I} \mid z)$ and $w_{5}(\mathcal{I} \mid z)$ are explicit. For the remaining solutions, we have $\alpha_{j}=-\frac{1}{2} \pi$ and $\beta_{j}=\frac{1}{2} \pi, j=2,3,4$. Accordingly, $w_{2}(\mathcal{I} \mid z), w_{3}(\mathcal{I} \mid z)$, and $w_{4}(\mathcal{I} \mid z)$ are implicit. Next, as $z \rightarrow \infty$ on ph $z=0$, there is no exponential dominance among the five solutions, and by applying Theorems 3.2 and 4.2 , we conclude that condition (1.8b), with $\mathcal{T}$ taken to be ph $z=0$, determines $w_{j}(\mathcal{I} \mid z)$ uniquely in all cases; in particular, for the overall dominant solution $w_{3}(\mathcal{I} \mid z)$ on $\mathrm{ph} z=0$. It also follows from the Lemma of $\S 2$ that if we were to modify equation (5.1) in such a way that the $\lambda$ 's are unchanged, but the $\mu$ 's all have equal real parts, then the weaker condition (1.8a) can be substituted for $(1.8 \mathrm{~b})$.

\section{Conclusions}

We have shown that at an irregular singularity of rank 1 , with unequal characteristic values, an explicit solution of the differential equation is always defined uniquely by its asymptotic expansion along any ray in the closure of the recessive sector of the solution. For an implicit solution, the asymptotic behavior needs to be maintained on two properly chosen rays, in general, in order to specify the solution uniquely.

Acknowledgment. The author is indebted to A. B. Olde Daalhuis and the referee for helpful comments.

\section{References}

1. D. Lutz and R. Schäfke, On the remainders of the asymptotic expansion of solutions of differential equations near irregular singular points, Complex Variables Theory Appl. 26 (1994), 203-212.

2. A. B. Olde Daalhuis, Hyperasymptotic solutions of higher order linear differential equations with a singularity of rank one, Proc. Roy. Soc. London, Ser. A 454 (1998), 1-29.

3. A. B. Olde Daalhuis and F. W. J. Olver, On the asymptotic and numerical solution of linear ordinary differential equations, SIAM Review 40 (1998), 463-495. 
4. F. W. J. Olver, Asymptotics and Special Functions, Academic Press, New York, 1974. Reprinted by AK Peters, Wellesley, 1997.

5. __ Asymptotic solutions of linear ordinary differential equations at an irregular singularity of rank unity, Methods Appl. Anal. 4 (1997), 375-403.

6. W. Wasow, Asymptotic Expansions for Ordinary Differential Equations, Wiley (Interscience), New York, 1965. Reprinted by Krieger, Huntington, 1976, and Dover, New York, 1987.

Institute for Physical Science and Technology and Department of Mathematics, UniverSity OF MARYland, College PaRK, MARYland 20742

E-mail: olver@bessel.umd.edu 